\title{
The development of the adult intestinal stem cells: Insights from studies on thyroid hormone- dependent amphibian metamorphosis
}

\author{
Yun-Bo Shi ${ }^{{ }^{*}}$, Takashi Hasebe ${ }^{2}$, Liezhen Fu' ${ }^{1}$ Kenta Fujimoto ${ }^{1}$ and Atsuko Ishizuya-Oka ${ }^{2^{*}}$
}

\begin{abstract}
Adult organ-specific stem cells are essential for organ homeostasis and repair in adult vertebrates. The intestine is one of the best-studied organs in this regard. The intestinal epithelium undergoes constant self-renewal throughout adult life across vertebrates through the proliferation and subsequent differentiation of the adult stem cells. This self-renewal system is established late during development, around birth, in mammals when endogenous thyroid hormone (T3) levels are high. Amphibian metamorphosis resembles mammalian postembryonic development around birth and is totally dependent upon the presence of high levels of T3. During this process, the tadpole intestine, predominantly a monolayer of larval epithelial cells, undergoes drastic transformation. The larval epithelial cells undergo apoptosis and concurrently, adult epithelial stem/progenitor cells develop de novo, rapidly proliferate, and then differentiate to establish a trough-crest axis of the epithelial fold, resembling the cryptvillus axis in the adult mammalian intestine. We and others have studied the T3-dependent remodeling of the intestine in Xenopus laevis. Here we will highlight some of the recent findings on the origin of the adult intestinal stem cells. We will discuss observations suggesting that liganded T3 receptor (TR) regulates cell autonomous formation of adult intestinal progenitor cells and that T3 action in the connective tissue is important for the establishment of the stem cell niche. We will further review evidence suggesting similar T3-dependent formation of adult intestinal stem cells in other vertebrates.
\end{abstract}

\section{Introduction}

Organ-specific adult stem cells are essential for the development of adult organs and tissue repair and regeneration. While most vertebrates develop directly into the adult form by birth, their organ development often involves a two-step process, the formation of an immature but often functional organ during embryogenesis followed by the maturation into the adult form. This second step takes place during the so-called post-embryonic development, a period around birth in mammals such as human and mouse when plasma thyroid hormone (T3) concentrations are high [1]. The organ-specific adult stem cells are often

\footnotetext{
* Correspondence: shi@helix.nih.gov; a-oka@nms.ac.jp

${ }^{1}$ Section on Molecular Morphogenesis, Laboratory of Gene Regulation and Development, Program in Cellular Regulation and Metabolism (PCRM), Eunice Kennedy Shriver National Institute of Child Health and Human Development (NICHD), National Institutes of Health $(\mathrm{NIH})$, Bethesda,

Maryland, 20892, USA

2Department of Biology, Nippon Medical School, Kawasaki, Kanagawa 2110063, Japan

Full list of author information is available at the end of the article
}

formed/matured during this period. One of the wellstudied such organs is the intestine. The tissue responsible for the main physiological function of the intestine, the intestinal epithelium, which is responsible for the food processing and nutrient absorption, is continuously renewed throughout adult life in vertebrates. This takes place through stem cell divisions in the crypt, followed by their differentiation as the cells migrate up to and along the villus and eventual death of the differentiated cells near the tip of the villus. In adult mammals, the intestinal epithelium is replaced once every 1-6 days [2-4], and in amphibians, this occurs in 2 weeks [5]. Such a self-renewal system has been shown to be present throughout vertebrates, from zebrafish, frogs, to human. While a number of signaling pathways have been shown to be important for intestinal development and cell renewal in the adult $[4,6]$, much less is known about how adult stem cells are formed during development, in part due to the difficulties to study the uterus-enclosed mammalian embryogenesis.
C Biomed Central

() 2011 Shi et al; licensee BioMed Central Ltd. This is an Open Access article distributed under the terms of the Creative Commons Attribution License (http://creativecommons.org/licenses/by/2.0), which permits unrestricted use, distribution, and reproduction in any medium, provided the original work is properly cited. 
Intestinal remodeling during amphibian metamorphosis offers a unique opportunity to study the development of adult organ-specific stem cells in vertebrates. As during postembryonic development in mammals, T3 levels in the plasma are high during amphibian metamorphosis. In fact, T3 is both necessary and sufficient for premetamorphic tadpoles to transform into frogs $[7,8]$. In premetamorphic tadpoles, there is little T3. The synthesis of endogenous T3 around stage 55 in Xenopus laevis initiates metamorphosis. The plasma T3 rises to peak levels at the climax of metamorphosis and subsequently is reduced to much lower levels by the end of metamorphosis. During metamorphosis, different organs undergo vastly different changes, including total resorption such as the tail and gills, de novo development such as the limb, and drastic remodeling such as the liver, pancreas and intestine, which involve both larval cell death and adult cell development. Despite such complex changes, all these changes are controlled by T3. An important advantage of this system is that it occurs independent of maternal influence as in the case of mammals. Furthermore, this process can be induced even in organ cultures of premetamorphic tadpoles when treated with physiological concentrations of T3 $[7,8]$. This makes it easy to manipulate and study the development and regulation of the adult organ-specific stem cells.

In the South African clawed toad Xenopus laevis, the tadpole intestine consists of largely a monolayer of larval epithelial cells, with thin layers of surrounding connective tissue and muscles [9] (Figure 1). When T3 becomes available either endogenously or exogenously, the vast majority, if not all, of the larval epithelial cells undergo apoptosis and concurrently, adult epithelial stem/progenitor cells appear de novo and rapidly proliferate [9-11](Note that organ-specific stem cells are traditionally defined for adult organs. It is possible that the developing stem cells during organogenesis are not the same as in adult organs. For simplicity, we will refer the proliferating adult epithelial cells with detectable stem cell markers during metamorphosis to as stem cells while those lacking the stem cell markers as progenitors). These adult epithelial cells subsequently differentiate to establish a trough-crest axis of epithelial folds by the end of metamorphosis, resembling the crypt-villus axis in the adult mammalian intestine, accompanied by the maturation of the connective tissue and muscles [9]. Just like all other processes during metamorphosis, all these changes during intestinal remodeling are controlled by T3 and can be induced even in organ cultures treated with T3 $[10,12,13]$, indicating organ-autonomous formation of adult stem/progenitor cells. Such interesting properties have allowed studies to determine the origin of the stem cells and investigate the mechanisms governing the formation of the adult stem cells.

\section{Mechanism of the regulation of Xenopus development by T3}

T3 binds to T3 receptors (TRs) with high affinities. TRs are transcription factors that form heterodimers with 9-cis retinoic acid receptors (RXRs) and these dimers bind to T3 response element (TRE) in/around the promoters of T3 target genes [14-17]. For T3-inducible genes, TR/RXR functions as a repressor in the absence of T3 and as an activator in the presence of T3. TRs recruit different cofactor complexes to TREs to affect transcription [15,18-25]. Extensive molecular and genetic studies by a number of different laboratories have shown that TR is both necessary and sufficient to mediate the metamorphic effects of T3 and that TR has dual functions during Xenopus development [26-35]. In premetamorphic Xenopus laevis tadpoles, TR recruits corepressor complexes to target genes when T3 is absent and this recruitment is important to keep the T3-inducible genes repressed, thus regulating metamorphic timing (Figure 1) [31,36-38]. After stage 55, when endogenous T3 becomes available, corepressor complexes are released and coactivator complexes are recruited by TR, this leads to the activation of T3 target genes and metamorphosis [33,34,39-43].

\section{The origin of the adult intestinal epithelial stem cells}

Earlier microscopic and cytological studies of intestinal remodeling in Xenopus laevis failed to identify any adult stem cells in premetamorphic tadpoles [9]. On the other hand, the ability to induce intestinal remodeling in intestinal organ cultures $[10,12,13]$ indicates that the adult stem cells develop de novo within tadpole intestine. It is possible that non-epithelial cells may give rise to the stem cells when T3 becomes available. Alternatively, some differentiated larval epithelial cells may undergo dedifferentiation to become the stem cells during metamorphosis. This latter scenario is supported by chronological observations of intestinal metamorphosis [44] and the apparent presence of the proteins of the differentiated epithelial cells in proliferating adult progenitor cells $[11,45]$. On the other hand, there has been no direct evidence to demonstrate the origin of the adult epithelial cells. Making use of a transgenic Xenopus line ubiquitously expressing the green fluorescent protein (GFP), we have recently carried out recombinant organ culture studies to determine the orgin of the adult intestinal epithelial stem cells [10].

As diagramed in Figure 2A, a fragment of the small intestine near the bile duct was isolated from GFP-expressing transgenic as well as wild type premetamorphic tadpoles. The epithelium and non-epithelial tissues, which are made of mainly the fibroblasts in the connective tissue, can be enzymatically separated from each other. The transgenic and wild type tissues were recombined into 4 types of 


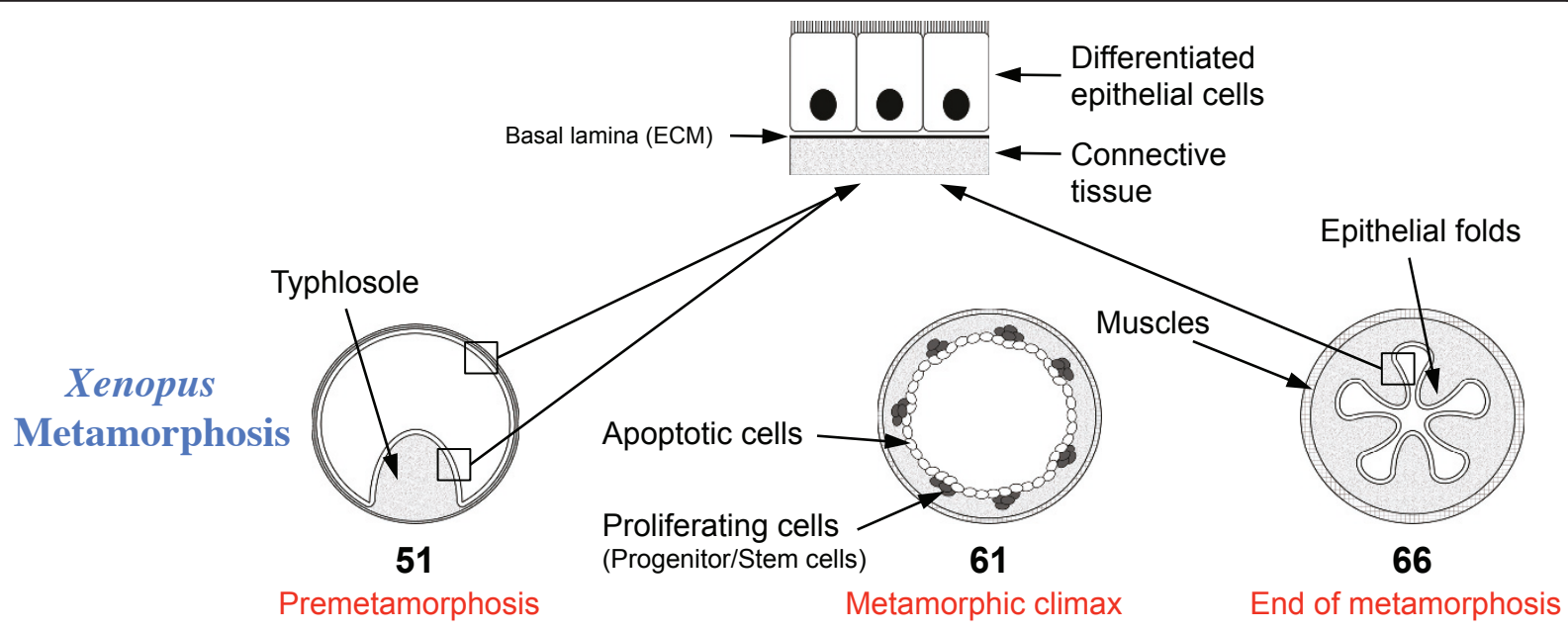

\section{T3-regulation of Metamorphosis}
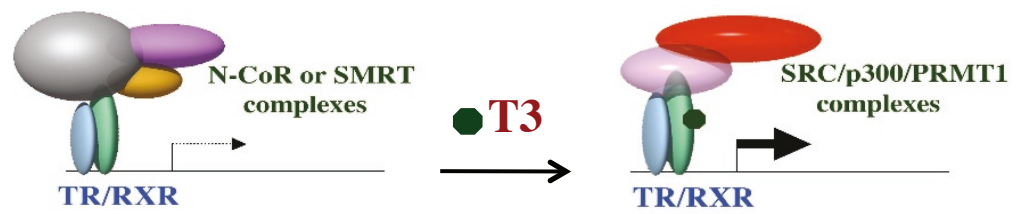

Figure 1 T3-dependent intestinal remodeling during Xenopus laevis metamorphosis serves as a model to study adult organ-specific stem cell development in vertebrates. Top: In premetamorphic tadpoles, there is little or no T3 and the intestine has a simple structure with only a single fold, the typhlosole. This structure is similar to the mammalian embryonic intestine. At the metamorphic climax when T3 level is high, the larval epithelial cells begin to undergo apoptosis, as indicated by the circles. Concurrently, the proliferating adult progenitor/stem cells develop de novo from larval epithelial cells through dedifferentiation, as indicated by black dots. By the end of metamorphosis, the newly differentiated adult epithelial cells form a multiply folded epithelium, similar to mammalian adult intestines. This process is entirely controlled by T3 and can be induced even in organ cultures of tadpole intestine with T3 treatment. In mammals, the intestine undergoes postembryonic maturation into the adult form around or shortly after birth when T3 levels are also high. Thus, intestinal metamorphosis offers a unique opportunity to study the development of adult intestinal stem cells. Bottom: T3 functions by regulating gene transcription through TRs. In the absence of T3 (as in premetamorphic tadpole), TR forms heterodimers with RXR and the heterodimer binds to target gene promoters to repress their expression by recruiting corepressor complexes containing the related proteins N-CoR or SMRT and histone deacetylases. When T3 is present, the corepressor complexes are released upon T3 binding to TR, and simultaneously coactivator complexes such as those containing SRC, p300, and PRMT1, are recruited to activate target gene expression. SRC and p300 are histone acetyltransferases and PRMT1 is a histone methyltransferase. The coactivator complexes will modify histones and activate gene expression to induce metamorphosis.

recombinant intestinal organ cultures and treated with a physiological concentration of T3, which induced intestinal remodeling just like in intact animals, namely, larval epithelial cell death and adult epithelium development. In all recombinant intestines, adult progenitor cells expressing markers for intestinal progenitor/stem cells such as sonic hedgehog (Shh) became detectable and then differentiated into the adult epithelium expressing intestinal fatty acid binding-protein (IFABP), a marker for absorptive cells. Importantly, whenever the epithelium was derived from transgenic intestine, both the adult progenitor/stem cells and differentiated cells expressed GFP, while neither of them expressed GFP if the epithelium was derived from wild type tadpoles [10]. These results thus demonstrate that the intestinal progenitor/stem cells that give rise to the adult intestinal epithelium originate from the larval epithelium, likely through T3-induced dedifferentiation of some larval epithelial cells.
Distinct roles of the epithelium and connective tissue in stem cell formation

Earlier organ culture studies have shown that adult epithelium formation requires the presence of the connective tissue [46]. How the connective tissue participates in this process remains largely unknown. Given that the adult epithelial stem cells originate from the larval epithelium, it is possible that T3 induces the formation of the stem cells in a cell-autonomous manner. To investigate whether T3 signaling in the epithelium alone is sufficient for adult stem cell formation, we have carried out recombinant organ culture experiments by using premetamorphic transgenic Xenopus laevis tadpoles that express a dominant positive TR (dpTR, which is constitutively active but does not bind to T3) under the control of a heat shock inducible promoter [29,47] (Figure 2A). Heat shock treatment of the recombinant intestinal organ cultures leads to the expression of dpTR only in 


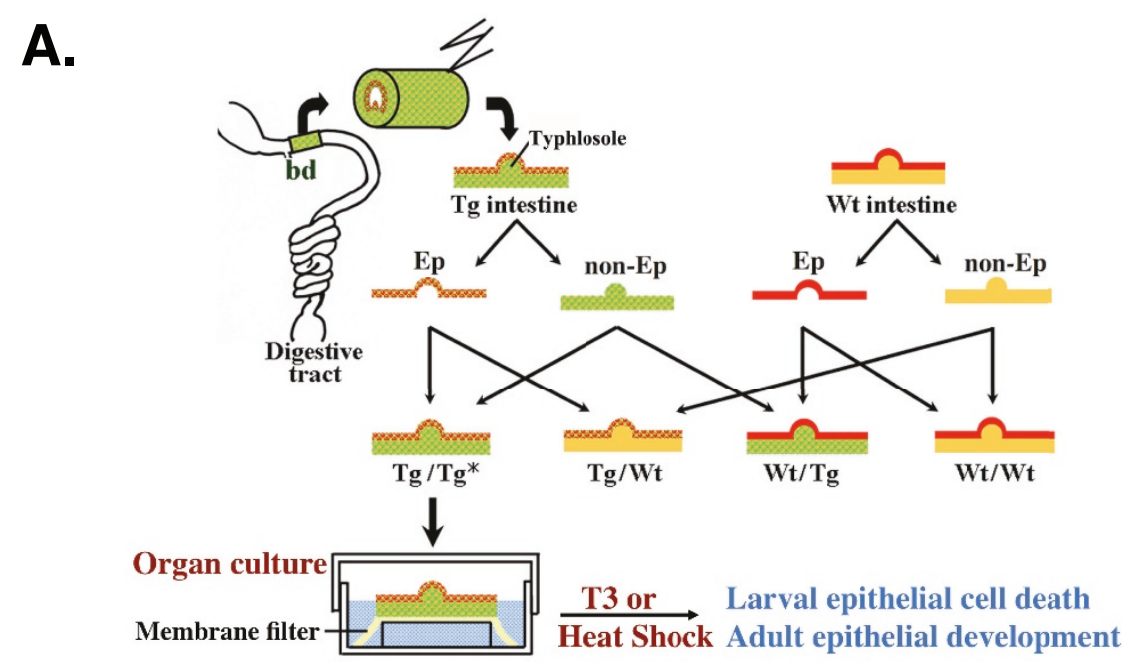

B.

\section{Ep/non-Ep \\ Progenitor marker}

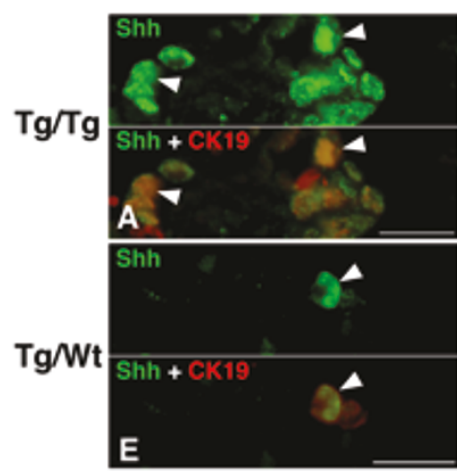

Stem cell markers

Msi1

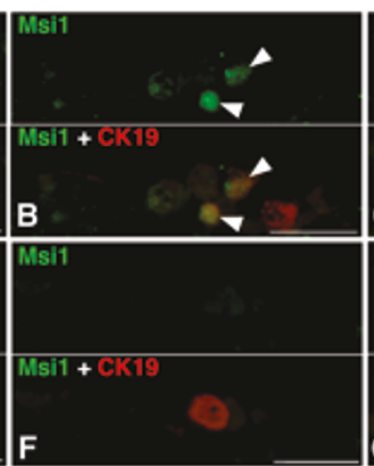

Akt
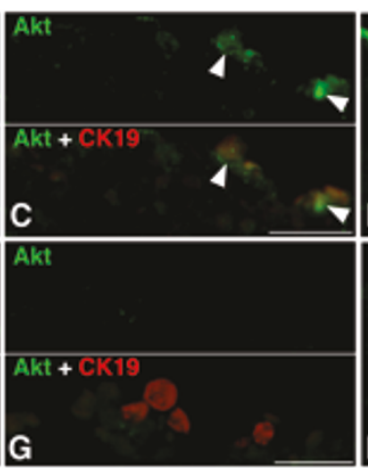

\section{Differentiation marker} IFABP

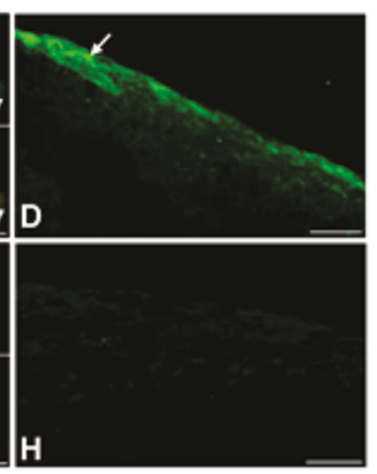

Figure 2 Recombinant intestinal organ culture studies using transgenic tadpole. A. Schematic diagram for tissue recombination and organ culture of the Xenopus laevis intestine. Transgenic (Tg) frogs are generated using a double promoter construct [61] where one GFP is expressed in the lens of the eye under the control of the $\gamma$-crystallin gene promoter for identification of the transgenic animals and another GFP is expressed ubiquitously under the CMV promoter or a dominant positive thyroid hormone receptor (dpTR, which resembles T3-bound TR, or activated TR, but does not need T3) is expressed under the control of a heat shock inducible promoter. The adult Tg frogs are used to produce $\mathrm{Tg}$ and wild type $(\mathrm{Wt})$ sibling tadpoles. Tubular fragments are isolated from the small intestine just behind the bile duct junction (bd) of premetamorphic (when there is little endogenous T3 present) Tg and Wt tadpoles, slit open lengthwise, and separated into epithelium (Ep) and non-epithelial tissues (non-Ep), which consists of mainly the connective tissue underlying the epithelium. Each Ep is then recombined with homologous or heterologous non-Ep. The four kinds of recombinant intestines are placed on membrane filters on grids and cultured in vitro. Heat shock treatment, when using dpTR tadpoles, or T3 treatment, when using GFP animals, is applied to induce intestinal remodeling. * indicated type of Ep/type of non-Ep used in the recombinant organ culture: e.g., Tg/Wt indicating $\mathrm{Tg}$ epithelium recombined with Wt nonepithelial tissues. B. Recombinant organ culture studies using dpTR-transgenic and Wt tadpoles indicate that only when both the Ep and non-Ep were derived from $\mathrm{Tg}$ animals, i.e., $\mathrm{Tg} / \mathrm{Tg}$, were true stem cells and adult epithelium formed after heat shock treatment. Recombinants made of $\mathrm{Tg} E P$ and $\mathrm{Tg}$ non-EP $(\mathrm{Tg} / \mathrm{Tg}$ ) (A-D) and $\mathrm{Tg}$ Ep and Wt non-EP (Tg/Wt) (E-H) of the intestines were cultured with heat shock treatment for $5(A-C, E-G)$ or $7(D, H)$ days in vitro. Cross sections were double-immuno-stained with anti-Shh (green, sonic hedgehog, an adult progenitor cell marker) and anti-CK19 (red, cytokeratin-19, which is expressed in epithelial cells. (A, E)), anti-Msi-1 (green, Musashi-1, a stem cell marker of the vertebrate adult intestine) and anti-CK19 (red; (B, F)), or anti-Akt (green, a stem cell marker of the vertebrate adult intestine) and anti-CK19 (red) antibodies (C, G), or immunostained with anti-IFABP antibody (green; D, H). In both Tg/Tg (arrowheads; (A)) and Tg/Wt intestines (E), cells positive for Shh become detectable on day 5 among cells expressing CK19, indicating the adult progenitor cells can be induced by cell-autonomous action of activated TR in the epithelium. Cells positive for Msi1 and Akt are also detected among CK19immunoreactive cells in Tg/Tg intestine (arrowheads; $(B, C)$ ) but not in Tg/Wt intestine ( $F, G)$. In addition, differentiated epithelial cells expressing IFABP were present after extended culturing only in $\mathrm{Tg} / \mathrm{Tg}$ organ cultures (D). Thus, activation of TR in the non-epithelial tissues is also required for the stem cell formation, likely by contributing to the formation of the stem cell niche. Not shown here is that most of the epithelial cells undergo apoptosis when dpTR is expressed in either the EP or non-EP or both, just like that during metamorphosis when T3 binds to TR. See [47] for details. 
the transgenic but not wild type tissues while the endogenous TR remains unliganded regardless of the treatment. When either the epithelium or the non-epithelial tissues or both were derived from the transgenic tadpoles, larval epithelial cell apoptosis could be detected after heat shock treatment for 5 days. On the other hand but expectedly, no metamorphic changes were observed after the heat shock treatment when both the epithelium and non-epithelial tissues were from the wild type animals [47]. Thus, larval epithelial cell death can occur via two pathways: suicide cell-autonomously through T3 action in the epithelial cells versus murder through T3 action in the non-epithelial tissues, which likely involves the induction of matrix metalloproteinases such as stromelysin-3, an MMP secreted by fibroblasts that causes epithelial cell death [48].

Adult epithelial progenitor cells expressing Shh were also detectable after 5 days of heat shcok treatment if the recombinant was made of epithelium derived from transgenic intestine, regardless of the origin of the non-epithelial tissues [47]. However, no such cells were present if both tissues were derived from the wild type animals or when the epithelium was from the wild type animals and the non-epithelial tissues were from the transgenic animals. Thus, TR activation in the non-epithelial tissues is not sufficient to induce the formation of the Shh+ adult progenitor cells in the epithelium, although it can induce epithelial apoptosis. The formation of the Shh+ progenitor cells appears to be cell-autonomous upon activation of TR (due to T3 binding or the expression of a constitutively active TR) in the epithelium [47].

When the expression of two stem cell markers of the adult mammalian intestine, Musashi-1 (Msi-1) and Akt [49-51], were analyzed after 5 days of heat shock treatment, they were detected in the Shh+ cells when both of the epithelium and non-epithelial tissues used to make the recombinant intestine were derived from dpTR-transgenic tadpole intestine (Figure 2B). Upon prolonged culturing ( 5 days of heat shock treatment followed by 2 days of culture without heat shock treatment), such organ cultures formed adult epithelium expressing IFABP, a marker for the absorptive epithelium (Figure 2B). On the other hand, if only the epithelium was derived from the transgenic tadpoles, the Shh+ progenitor cells produced after heat shock treatment failed to express either of the stem cell markers and failed to develop into differentiated adult epithelium expressing IFABP (Figure 2B). These results indicate that while the adult progenitor/stem cells can be induced by T3 in the larval epithelium, liganded TR-mediated gene expression in the surrounding tissues other than the epithelium is required for them to develop into adult stem cells, suggesting that T3 activation of the non-epithelial tissues, most likely the underlying connective tissue is required for the establishment of the stem cell niche in the amphibian intestine during metamorphosis. Consistently, an earlier transgenic study where a dominant negative TR was expressed under the control of epithelial-, fibroblast-, and muscle-specific gene promoters showed that while mutant TR expression in different tissues caused distinct phenotypes in the postmetamorphic intestine, its expression in either the epithelium and fibroblasts led to abnormal epithelia and mesenchyme development [35], supporting our conclusion that TR activation in both the epithelium and connective tissue is important for adult epithelium development.

\section{A role of the TR-coactivator protein arginine methyltransferase 1 (PRMT1) in stem cell formation during metamorphosis by $\mathrm{T} 3$}

As described above, TR functions by recruiting coactivator complexes in the presence of T3 to induce gene regulation and metamorphosis. Earlier studies have shown that the coactivator complexes containing SRC3, p300, and PRMT1, are essential for metamorphosis in Xenopus laevis [39-43]. Interestingly, the expression of PRMT1 is upregulated in the intestine during metamorphosis. Kinetic studies of the induction of PRMT1 expression by T3 suggest that it is indirectly regulated by TR. In situ hybridization analysis have revealed that it is first upregulated in a small number of larval epithelial cells at early stages of metamorphosis when T3 levels are still low and that these cells appear to dedifferentiate into adult stem cells $[52,53]$. More importantly, in transgenic tadpoles overexpressing PRMT1 under a heat shock inducible promoter, heat shock treatment led to an increase in the number of progenitor/stem cells in the intestine during natural metamorphosis [53]. On the other hand, knocking down the expression of endogenous PRMT1 with an antisense morpholino oligonucleotide reduced the number of such cells during T3-induced metamorphosis (Figure 3) [53]. Thus, PRMT1 likely plays a role in the formation and/or proliferation of the intestinal progenitor/stem cells, possibly via its coactivator function for liganded TR.

\section{Evolutionary conservation in adult intestinal stem cell development}

The intestine is physiologically and structurally conserved in adult vertebrates, with the proliferating stem cells located in the crypts/troughs of the epithelial folds and differentiated epithelial cells located along the villi or the folds. At the tip of the villus or fold, the differentiated epithelial cells degenerate through apoptosis. The maturation of this adult structure occurs at different stage of development in different animals but interestingly all occurring around the time when plasma T3 levels are high, just like during amphibian metamorphosis. T3 or TR deficiency leads to abnormal intestinal morphology, a decrease in the number of epithelial cells along the crypt-villus axis 
A.
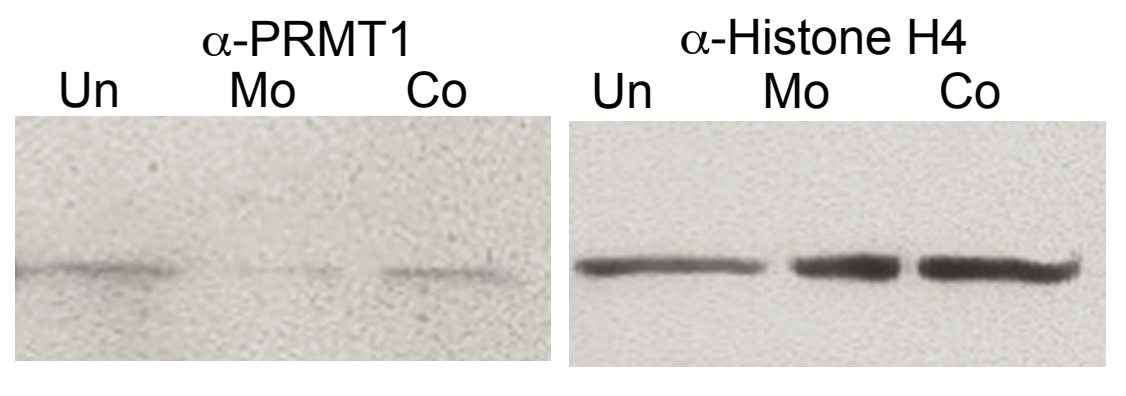

B.

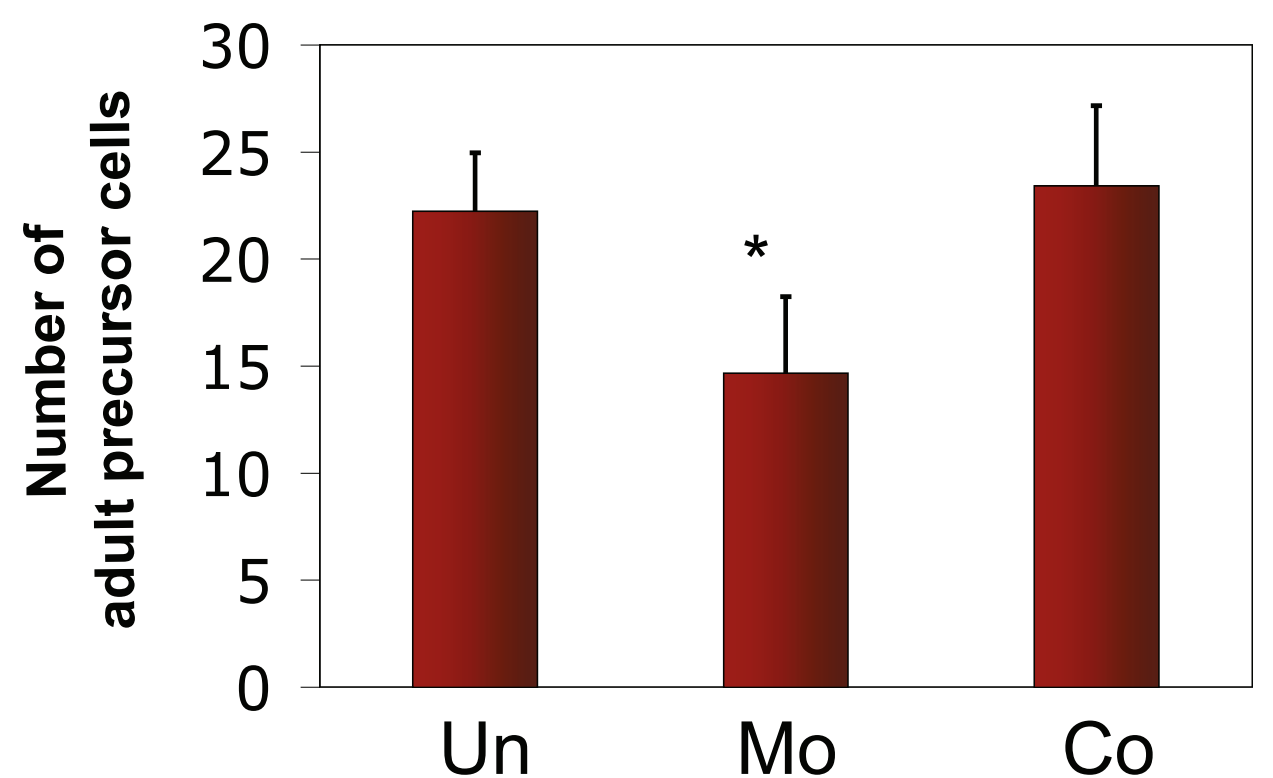

Figure 3 PRMT1 is required for the development and/or proliferation of adult intestinal progenitor/stem cells. A. In vivo knock-down of PRMT1 expression. Xenopus laevis tadpoles at stage 53/54 were injected with (MO, Co) or without (Un) PRMT1 (MO) or control (Co) antisense Vivo-morpholino oligos for 4 consecutive days. Intestines were dissected for western blot analysis of the expression of the endogenous PRMT1 or histone H4 (as a control). B. Knocking-down the expression of endogenous PRMT1 results in the reduction in the number of proliferating adult cells in the intestine. Stage 53/54 premetamorphic tadpoles were injected without (untreated) or with PRMT1 (MO) or control Vivo-morpholino oligos for four days. All tadpoles were then treated with $5 \mathrm{nM}$ of thyroid hormone for 4 days. The tadpoles were sacrificed and the intestine was analyzed for the number of proliferating adult cells. See [53] for details.

and in proliferating crypt cells [54-57]. It has been shown that TR $\alpha 1$ controls intestinal development during maturation at weaning as well as intestinal homeostasis in adulthood through the activation of the proliferation of intestinal progenitors by liganded TR $\alpha 1$ [58]. Thus, T3 and TR appear to have conserved roles in regulating the formation of the adult intestine in mouse and frog. Interestingly, PRMT1 has a conserved spatiotemporal expression pattern during postembryonic intestinal development in vertebrates. During mosue and zebrafish development, little PRMT1 expression was detected in the larval/neonatal intestine in zebrafish or mouse when plasma T3 levels were low (Figure 4A, D). During the transition to the adult intestine when T3 levels were high $[59,60]$, high levels of
PRMT1 mRNA were present but only in the bottom of the developing epithelial fold in zebrafish (Figure 4B) or the crypt of the mouse intestine (Figure 4E). In adult animals, the PRMT1 expression was high only in the mouse crypts and the bottom of the zebrafish intestinal epithelial folds, where the stem cells were located (Figure 4C, F). These results suggest that like during Xenopus metamorphosis, T3 likely regulates the development of the adult epithelial progenitor/stem cells in zebrafish and mouse intestine and that PRMT1 plays a role in this process. They also suggest that although neonatal mouse intestine is structurally fully developed, with the proper crypt-villus organization similar to that in the adult mouse, the embryonic/neonatal mouse intestinal stem cells are molecularly distinct from those in 


\section{Thyroid hormone}

very low

Larval/Neonatal

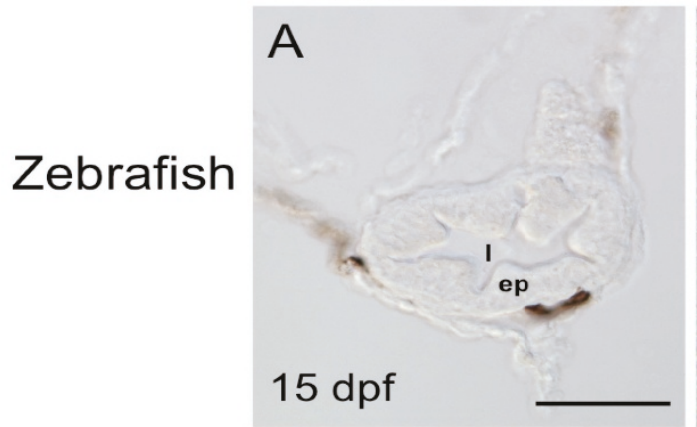

Mouse

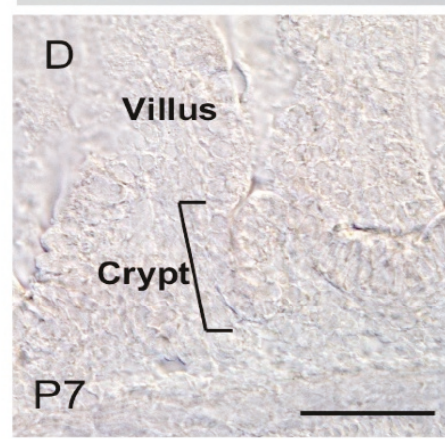

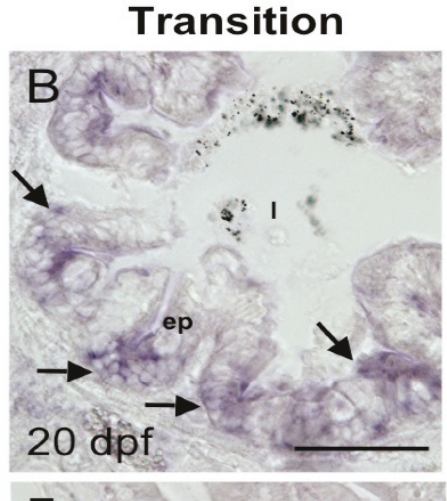

high

Transition

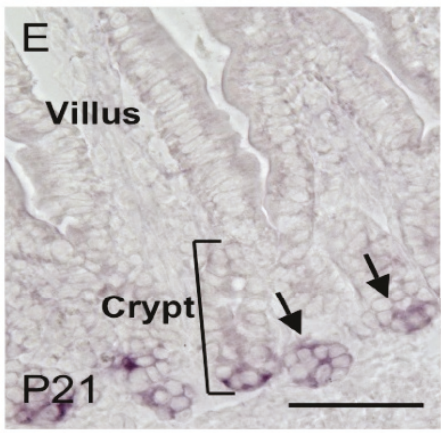

Iow
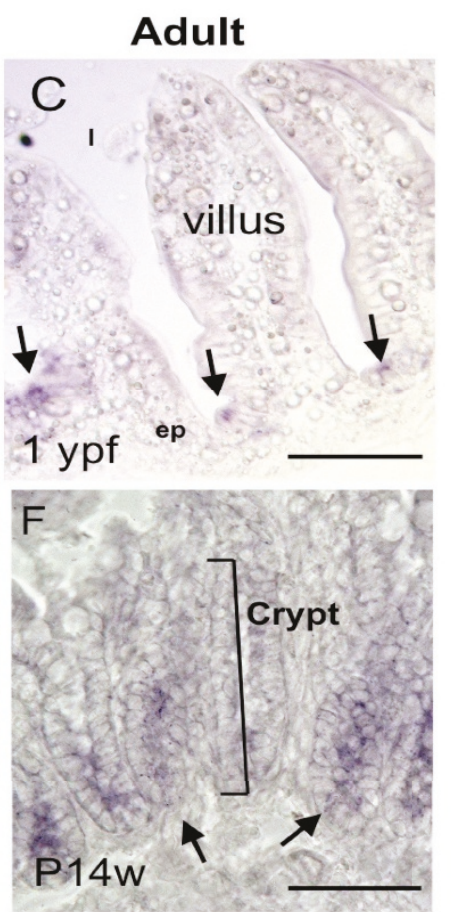

Figure 4 Conserved spatiotemporal expression patterns of PRMT1 in the postembryonic intestines of fish and mouse suggest a conserved role of PRMT1 in adult intestinal stem cell development. PRMT1 mRNA was analyzed by in situ hybridization in the intestines of zebrafish at 15 days post fertilization (dpf) (A), $20 \mathrm{dpf}$ (B) and 1 year post fertilization (ypf) (C) and mouse at postnatal day 7 (D, P7), P21 (E), and postnatal week 14 (F, P14w). Arrows indicate PRMT1 positive cells in the intestinal epithelium. Note that high levels of PRMT1 expression is detected only in the proliferating/stem cells located in the crypts in both species, resembling that in Xenopus laevis. In addition, the transition period in both species when PRMT1 is upregulated is characterized by high levels of T3 in the serum, just like that during Xenopus

metamorphosis. ep, epithelium. I, lumen. Bars, 50 mm. See [53] for details.

the adult mouse intestine. Thus, the formation of the adult intestinal stem cells may utilize conserved mechanisms, such as dependence on T3 and involvement of PRMT1, during vertebrate development.

\section{Conclusion}

Organ-specific adult stem cells play an essential role in organ homeostasis and tissue repair and regeneration. Understanding the mechanisms of their development is undoubtedly important for the generation and utilization of such cells for tissue replacement therapies and prevention of stem cell-related diseases. The uterus-enclosed development of mammalian embryos has hindered the study of the development of adult organ-specific stem cells in mammals. On the other hand, the conservation of the organ function and development makes it possible to use non-mammalian models for such purpose. The intestine is such an organ that is structurally and physiologically conserved across vertebrate species. Using Xenopus laevis metamorphosis as a model, we and others have shown that the adult intestinal stem cells develop de novo and are distinct molecularly from the larval cells. It is interesting to note that larval epithelial cells in premetamorphic tadpole intestines are mitotically active, even though they are differentiated and serve the physiological function of the intestinal epithelium [5]. Thus, to develop into stem cells, the larval epithelial cells merely need to repress the expression of the genes associated with differentiated cells and escape the T3-induced apoptotic fate that most other epithelial cells have. Intestinal organ culture studies suggest that T3 induces the epithelium to undergo cell-autonomous formation of adult progenitor cells and also induces the non-epithelial tissues, most likely the connective tissue, to form or contribute to the establishment of the adult stem cell niche. It is possible that some of the larval epithelial cells, in the context of the stem cell niche formed with the participation of the T3activated connective tissue, adopt the pathway of stem cell development during metamorphosis. The vast majority of the larval epithelial cells undergo apoptosis due to the lack 
of the proper stem cell niche environment even though they also lose the expression of at least some differentiation markers such as IFABP. The studies with PRMT1 have not only revealed an important role of this TR-coactivator in adult stem cell development/proliferation but also provided further evidence that adult stem cell development utilize conserved mechanisms across vertebrates.

\section{Acknowledgements}

This research was supported in part by the Intramural Research Program of $\mathrm{NICHD}, \mathrm{NIH}$ and the JSPS Grants-in-Aid for Scientific Research (C) (Grant number 20570060 to A. I.-O.).

\section{Author details \\ 'Section on Molecular Morphogenesis, Laboratory of Gene Regulation and Development, Program in Cellular Regulation and Metabolism (PCRM), Eunice Kennedy Shriver National Institute of Child Health and Human Development (NICHD), National Institutes of Health $(\mathrm{NIH})$, Bethesda, Maryland, 20892, USA. ${ }^{2}$ Department of Biology, Nippon Medical School, Kawasaki, Kanagawa 211-0063, Japan.}

\section{Authors' contributions}

YBS, TH, and Al-O wrote the manuscript, LF and KF participated in the discussion and revision. All authors read and approved the final manuscript.

\section{Competing interests}

The authors declare that they have no competing interests.

Received: 20 June 2011 Accepted: 6 September 2011

Published: 6 September 2011

\section{References}

1. Tata JR: Gene expression during metamorphosis: an ideal model for post-embryonic development. Bioessays 1993, 15(4):239-248.

2. MacDonald WC, Trier JS, Everett NB: Cell proliferation and migration in the stomach, duodenum, and rectum of man: Radioautographic studies. Gastroenterology 1964, 46:405-417.

3. Toner PG, Carr KE, Wyburn GM: The Digestive System: An Ultrastructural Atlas and Review. London: Butterworth; 1971.

4. van der Flier LG, Clevers H: Stem Cells, Self-Renewal, and Differentiation in the Intestinal Epithelium. Annu Rev Physiol 2009, 71:241-260.

5. McAvoy JW, Dixon KE: Cell proliferation and renewal in the small intestinal epithelium of metamorphosing and adult Xenopus laevis. J Exp Zool 1977, 202:129-138.

6. Sancho E, Eduard Batlle E, Clevers H: Signaling pathways in intestinal development and cancer. Annu Rev Cell DevBiol 2004, 20:695-723.

7. Gilbert LI, Tata JR, Atkinson BG: Metamorphosis: Post-embryonic reprogramming of gene expression in amphibian and insect cells. New York: Academic Press; 1996.

8. Shi Y-B: Amphibian Metamorphosis: From morphology to molecular biology. New York: John Wiley \& Sons, Inc; 1999.

9. Shi Y-B, Ishizuya-Oka A: Biphasic intestinal development in amphibians: Embryogensis and remodeling during metamorphosis. Current Topics in Develop Biol 1996, 32:205-235.

10. Ishizuya-Oka A, Hasebe T, Buchholz DR, Kajita M, Fu L, Shi YB: Origin of the adult intestinal stem cells induced by thyroid hormone in Xenopus laevis. Faseb J 2009, 23:2568-2575.

11. Schreiber AM, Cai L, Brown DD: Remodeling of the intestine during metamorphosis of Xenopus laevis. Proc Natl Acad Sci USA 2005, 102(10):3720-3725

12. Ishizuya-Oka A, Shimozawa A: Induction of metamorphosis by thyroid hormone in anuran small intestine cultured organotypically in vitro. In Vitro Cell Dev Biol 1991, 27A(11):853-857.

13. Ishizuya-Oka A, Ueda S, Damjanovski S, Li Q, Liang VC, Shi Y-B: Anteroposterior gradient of epithelial transformation during amphibian intestinal remodeling: immunohistochemical detection of intestinal fatty acid-binding protein. Dev Biol 1997, 192(1):149-161.
14. Lazar MA: Thyroid hormone receptors: multiple forms, multiple possibilities. Endocr Rev 1993, 14(2):184-193.

15. Yen PM: Physiological and molecular basis of thyroid hormone action. Physiol Rev 2001, 81(3):1097-1142.

16. Mangelsdorf DJ, Thummel C, Beato M, Herrlich P, Schutz G, Umesono K, et al: The nuclear receptor superfamily: the second decade. Cell 1995, 83(6):835-839.

17. Tsai MJ, O'Malley BW: Molecular mechanisms of action of steroid/thyroid receptor superfamily members. Ann Rev Biochem 1994, 63:451-486.

18. Ito M, Roeder RG: The TRAP/SMCC/Mediator complex and thyroid hormone receptor function. Trends Endocrinol Metab 2001, 12(3):127-134

19. Rachez C, Freedman LP: Mechanisms of gene regulation by vitamin $D(3)$ receptor: a network of coactivator interactions. Gene 2000, 246(1-2):9-21.

20. Zhang J, Lazar MA: The mechanism of action of thyroid hormones. Annu Rev Physiol 2000, 62:439-466.

21. Burke LJ, Baniahmad A: Co-repressors 2000. FASEB J 2000, 14(13):1876-1888.

22. Jepsen K, Rosenfeld MG: Biological roles and mechanistic actions of corepressor complexes. J Cell Sci 2002, 115(Pt 4):689-698.

23. Jones PL, Shi Y-B: N-CoR-HDAC corepressor complexes: Roles in transcriptional regulation by nuclear hormone receptors. In Current Topics in Microbiology and Immunology: Protein Complexes that Modify Chromatin. Volume 274. Edited by: Workman JL. Berlin: Springer-Verlag; 2003:237-268.

24. Rachez C, Freedman LP: Mediator complexes and transcription. Curr Opin Cell Biol 2001, 13(3):274-280.

25. Hu X, Lazar MA: Transcriptional Repression by Nuclear Hormone Receptors. TEM 2000, 11(1):6-10.

26. Schreiber AM, Das B, Huang H, Marsh-Armstrong N, Brown DD: Diverse developmental programs of Xenopus laevis metamorphosis are inhibited by a dominant negative thyroid hormone receptor. PNAS 2001, 98:10739-10744.

27. Brown DD, Cai L: Amphibian metamorphosis. Dev Biol 2007, 306(1):20-33.

28. Buchholz DR, Hsia VS-C, Fu L, Shi Y-B: A dominant negative thyroid hormone receptor blocks amphibian metamorphosis by retaining corepressors at target genes. Mol Cell Biol 2003, 23:6750-6758.

29. Buchholz DR, Tomita A, Fu L, Paul BD, Shi Y-B: Transgenic analysis reveals that thyroid hormone receptor is sufficient to mediate the thyroid hormone signal in frog metamorphosis. Mol Cell Biol 2004, 24:9026-9037.

30. Buchholz DR, Paul BD, Fu L, Shi YB: Molecular and developmental analyses of thyroid hormone receptor function in Xenopus laevis, the African clawed frog. Gen Comp Endocrinol 2006, 145(1):1-19.

31. Shi Y-B: Dual functions of thyroid hormone receptors in vertebrate development: the roles of histone-modifying cofactor complexes. Thyroid 2009, 19:987-999.

32. Nakajima K, Yaoita Y: Dual mechanisms governing muscle cell death in tadpole tail during amphibian metamorphosis. Dev Dyn 2003, 227:246-255.

33. Denver RJ, Hu F, Scanlan TS, Furlow JD: Thyroid hormone receptor subtype specificity for hormone-dependent neurogenesis in Xenopus laevis. Dev Biol 2009, 326(1):155-168.

34. Bagamasbad P, Howdeshell KL, Sachs LM, Demeneix BA, Denver RJ: A role for basic transcription element-binding protein 1 (BTEB1) in the autoinduction of thyroid hormone receptor beta. J Biol Chem 2008, 283:2275-2285.

35. Schreiber AM, Mukhi S, Brown DD: Cell-cell interactions during remodeling of the intestine at metamorphosis in Xenopus laevis. Dev Biol 2009, 331(1):89-98

36. Tomita A, Buchholz DR, Shi Y-B: Recruitment of N-CoR/SMRT-TBLR1 corepressor complex by unliganded thyroid hormone receptor for gene repression during frog development. Mol Cell Biol 2004, 24:3337-3346.

37. Sachs LM, Jones PL, Havis E, Rouse N, Demeneix BA, Shi Y-B: N-CoR recruitment by unliganded thyroid hormone receptor in gene repression during Xenopus laevis development. Mol Cell Biol 2002, 22:8527-8538.

38. Sato Y, Buchholz DR, Paul BD, Shi Y-B: A role of unliganded thyroid hormone receptor in postembryonic development in Xenopus laevis. Mechanisms of Development 2007, 124:476-488

39. Matsuda H, Paul BD, Choi CY, Hasebe T, Shi Y-B: Novel functions of protein arginine methyltransferase 1 in thyroid hormone receptor-mediated transcription and in the regulation of metamorphic rate in Xenopus laevis. Mol Cell Biol 2009, 29:745-757. 
40. Paul BD, Buchholz DR, Fu L, Shi Y-B: Tissue- and gene-specific recruitment of steroid receptor coactivator-3 by thyroid hormone receptor during development. J Biol Chem 2005, 280:27165-27172.

41. Paul BD, Fu L, Buchholz DR, Shi Y-B: Coactivator recruitment is essential for liganded thyroid hormone receptor to initiate amphibian metamorphosis. Mol Cell Biol 2005, 25:5712-5724.

42. Paul BD, Buchholz DR, Fu L, Shi Y-B: SRC-p300 coactivator complex is required for thyroid hormone induced amphibian metamorphosis/. J Biol Chem 2007, 282:7472-7481.

43. Havis E, Sachs LM, Demeneix BA: Metamorphic T3-response genes have specific co-regulator requirements. EMBO Reports 2003, 4:883-888.

44. Marshall JA, Dixon KE: Cell proliferation in the intestinal epithelium of Xenopus laevis tadpoles. J Exp Zool 1978, 203:31-40

45. Amano T, Noro N, Kawabata H, Kobayashi Y, Yoshizato K: Metamorphosisassociated and region-specific expression of calbindin gene in the posterior intestinal epithelium of Xenopus laevis larva. Dev Growth Differ 1998, 40:177-188.

46. Ishizuya-Oka A, Shimozawa A: Connective tissue is involved in adult epithelial development of the small intestine during anuran metamorphosis in vitro. Roux's Arch Dev Biol 1992, 201:322-329.

47. Hasebe T, Buchholz DR, Shi YB, Ishizuya-Oka A: Epithelial-connective tissue interactions induced by thyroid hormone receptor are essential for adult stem cell development in the Xenopus laevis intestine. Stem Cells 2011, 29(1):154-161.

48. Fu L, Ishizuya-Oka A, Buchholz DR, Amano T, Matsuda H, Shi YB: A causative role of stromelysin-3 in extracellular matrix remodeling and epithelial apoptosis during intestinal metamorphosis in Xenopus laevis. J Biol Chem 2005, 280(30):27856-27865.

49. Hasebe T, Kajita M, Shi YB, Ishizuya-Oka A: Thyroid hormone-up-regulated hedgehog interacting protein is involved in larval-to-adult intestinal remodeling by regulating sonic hedgehog signaling pathway in Xenopus laevis. Dev Dyn 2008, 237(10):3006-3015.

50. Ishizuya-Oka A, Ueda S, Inokuchi T, Amano T, Damjanovski S, Stolow M, et al: Thyroid hormone-induced expression of Sonic hedgehog correlates with adult epithelial development during remodeling of the Xenopus stomach and intestine. Differentiation 2001, 69:27-37.

51. Ishizuya-Oka A, Shimizu K, Sakakibara S, Okano H, Ueda S: Thyroid hormone-upregulated expression of Musashi-1 is specific for progenitor cells of the adult epithelium during amphibian gastrointestinal remodeling. J Cell Sci 2003, 116(Pt 15):3157-3164.

52. Sun G, Hasebe T, Fujimoto K, Lu R, Fu L, Matsuda H, et al: Spatio-temporal expression profile of stem cell-associated gene LGR5 in the intestine during thyroid hormone-dependent metamorphosis in Xenopus laevis. PLoS One 2010, 5(10):e13605.

53. Matsuda H, Shi YB: An essential and evolutionarily conserved role of protein arginine methyltransferase 1 for adult intestinal stem cells during postembryonic development. Stem Cells 2010, 28(11):2073-2083.

54. Plateroti M, Gauthier K, Domon-Dell C, Freund JN, Samarut J, Chassande O: Functional interference between thyroid hormone receptor alpha (TRalpha) and natural truncated TRDeltaalpha isoforms in the control of intestine development. Mol Cell Biol 2001, 21(14):4761-4772.

55. Flamant F, Poguet AL, Plateroti M, Chassande O, Gauthier K, Streichenberger $\mathrm{N}$, et al: Congenital hypothyroid Pax8(-/-) mutant mice can be rescued by inactivating the TRalpha gene. Mol Endocrinol 2002, 16(1):24-32.

56. Kress E, Rezza A, Nadjar J, Samarut J, Plateroti M: The frizzled-related sFRP2 gene is a target of thyroid hormone receptor alpha1 and activates betacatenin signaling in mouse intestine. J Biol Chem 2009, 284(2):1234-1241.

57. Plateroti M, Chassande O, Fraichard A, Gauthier K, Freund JN, Samarut J, et al: Involvement of T3Ralpha- and beta-receptor subtypes in mediation of T3 functions during postnatal murine intestinal development. Gastroenterology 1999, 116(6):1367-1378.

58. Plateroti M, Kress E, Mori Jl, Samarut J: Thyroid hormone receptor alpha1 directly controls transcription of the beta-catenin gene in intestinal epithelial cells. Mol Cell Biol 2006, 26(8):3204-3214.

59. Brown DD: The role of thyroid hormone in zebrafish and axoloft development. Proc Natl Acad Sci USA 1997, 94:13011-13016.

60. Friedrichsen S, Christ S, Heuer H, Schäfer MKH, Mansouri A, Bauer K, et al: Regulation of iodothyronine deiodinases in the Pax8-/- mouse model of congenital hypothyroidism. Endocrinology 2003, 144:777-784.
61. Fu L, Buchholz D, Shi YB: Novel double promoter approach for identification of transgenic animals: A tool for in vivo analysis of gene function and development of gene-based therapies. Mol Reprod Dev 2002, 62(4):470-476.

doi:10.1186/2045-3701-1-30

Cite this article as: Shi et al.: The development of the adult intestinal stem cells: Insights from studies on thyroid hormone-dependent amphibian metamorphosis. Cell \& Bioscience 2011 1:30.

\section{Submit your next manuscript to BioMed Central and take full advantage of:}

- Convenient online submission

- Thorough peer review

- No space constraints or color figure charges

- Immediate publication on acceptance

- Inclusion in PubMed, CAS, Scopus and Google Scholar

- Research which is freely available for redistribution

Submit your manuscript at www.biomedcentral.com/submit
Biomed Central 